\title{
Steady-State Kinetic and Thermodynamic Analysis of the Reactions Catalyzed by Two Pantothenases from Pseudomonads
}

\author{
R. Kalervo Airas \\ Department of Biochemistry, University of Turku, SF-20500 Turku, Finland
}

\begin{abstract}
Airas, R. K., 1989. Steady-state Kinetic and Thermodynamic Analysis of the Reactions Catalyzed by Two Pantothenases from Pseudomonads. - Acta Chem. Scand. 43: 386-394.

A steady-state kinetic analysis of the pantothenase-catalyzed decomposition of pantothenic acid has been carried out by measuring the forward reaction rate, the pantothenate $-\beta$-alanine isotope exchange rate, the reverse-reaction rate, and the equilibrium constant, all at different substrate and product concentrations. The derived constants are the second-order rate constant, the apparent dissociation constant of $\beta$-alanine, the dissociation constant of pantoyl lactone, and the rate constants for the forward and reverse reactions. The procedure was repeated with two different pantothenases at different temperatures, the thermodynamic parameters were calculated and finally the $\Delta G, \Delta H$, and $\Delta S$ profiles of the pantothenasecatalyzed reaction were drawn. The results with both enzymes are consistent with an acyl enzyme mechanism where the acylation is at least ten times faster than the deacylation. There is a distinct difference in the $\Delta H$ value (and consequently in the $\Delta S$ value) derived from the temperature dependence of the apparent dissociation constant of $\beta$-alanine. In the pantoyl lactone-producing pantothenase the $\Delta H$ value is positive $\left(21 \mathrm{~kJ} \mathrm{~mol}^{-1}\right)$ but in the pantoic acid-producing pantothenase negative $\left(-12 \mathrm{~kJ} \mathrm{~mol}^{-1}\right)$. The difference can be partly explained by the participation of water in the latter enzyme reaction but not in the former.
\end{abstract}

Pantothenases (EC 3.5.1.22) are amidases from pseudomonads decomposing pantothenic acid. ${ }^{1,2} \beta$-Alanine is one product of these enzymes. A recent characterization of two such enzymes showed that one of them, the pantothenase from Pseudomonas fluorescens UK-1, produces pantoyl lactone while the product of another, pantothenase from Pseudomonas PS-21, is pantoic acid. ${ }^{3}$

There are some common kinetic features of the pantothenases. The rate of the pantothenate $-\beta-\left[{ }^{14} \mathrm{C}\right]$ alanine isotope-exchange reaction shows that the $-\mathrm{NH}_{2}$ form of the $\beta$-alanine participates in the reverse reaction, in a manner similar to the aminolytic deacylation of other amidases. $^{4,5}$

The existence of the isotope-exchange reaction in addition to the reverse reaction makes it possible to analyse some individual and combined rate constants even by steady-state methods. In this paper such an analysis of the kinetic constants was made at different temperatures with both of the pantothenases mentioned above, and the thermodynamic parameters were calculated for those rate and equilibrium constants which could be analyzed. One reason for the analysis was to check whether the difference in the mechanism is reflected by the thermodynamic parameters.

\section{Experimental}

Materials. The calcium pantothenate was from Merck (Darmstadt, FRG). Calcium ions were removed with an ion exchanger (Dowex 50), and the pantothenic acid was titrated with potassium hydroxide to $\mathrm{pH} 7$. The D-pantoyl lactone [(-)-2,4-dihydroxy-3,3-dimethyl-D-butyric acid $\gamma$ lactone] and $\beta$-alanine were from Fluka AG (Buchs, Switzerland). The D-pantoic acid potassium salt was prepared from the lactone by keepting the $\mathrm{pH}$ of the solution between $10-11$ by continuous additions of potassium hydroxide over about $2 \mathrm{~h}$ at room temperature. $\left[1-{ }^{14} \mathrm{C}\right]$ Pantothenate and $\beta-\left[1-{ }^{14} \mathrm{C}\right]$ alanine were from New England Nuclear (Dreieich, FRG).

The pantothenase from $P$. fluorescens UK-1 (NCIB 12017) and the pantothenase from Pseudomonas PS-21 were prepared as described earlier. ${ }^{2,3}$

Enzyme assays. Radioisotope-based methods using either $\left[{ }^{14} \mathrm{C}\right]$ pantothenate or $\beta-\left[{ }^{14} \mathrm{C}\right]$ alanine were used to measure the various pantothenase-catalyzed partial reactions. The details of the method have been described earlier. ${ }^{2}$

I. The rate of the transfer of radioactivity from pantothenate to $\beta$-alanine was measured in reaction mixtures of $100 \mu \mathrm{l}$ containing following five components: Potassium D-pantothenate (varied between $1-20 \mathrm{mM}$ ); D-[1- $\left.{ }^{14} \mathrm{C}\right]$ pantothenate, about $5000 \mathrm{~Bq}$ per $100 \mu \mathrm{l}$; buffer $(50 \mathrm{mM}$ Tris/12.5 $\mathrm{mM} \mathrm{H}_{2} \mathrm{SO}_{4}$, $\mathrm{pH} 8.1$ for pantothenase UK-1 and $25 \mathrm{mM}$ Tris/25 mM Tricin, $\mathrm{pH} 8.1$ for pantothenase PS-21); $\beta$-alanine $(0-30 \mathrm{mM})$; and the enzyme. A fixed time assay of $10 \mathrm{~min}$ was used, and the enzyme concentration was chosen such that it was low enough to give linear reactionprogress curves. The produced radioactive $\beta$-alanine was separated from pantothenate by paper chromatography in 
1-butanol/acetic acid/water (25:4:10, v/v). Tricin was used instead of $\mathrm{H}_{2} \mathrm{SO}_{4}$ to adjust the $\mathrm{pH}$ of the reaction mixtures of pantothenase PS-21, to avoid the sulphate inhibition of this enzyme. ${ }^{3}$ The $\mathrm{pH}$ of the buffers was not adjusted separately at different temperatures. The effect of the change in $\mathrm{pH}$ was assumed to be eliminated by the fact that the protonation of the ionizable groups affecting the enzymic activity (imidazole and amino groups) is approximately as dependent on temperature as the protonation of the buffers Tris and Tricin. ${ }^{6}$

II. The rate of the pantothenate $-\beta-\left[{ }^{14} \mathrm{C}\right]$ alanine isotope-exchange reaction was measured in the same way as the forward reaction above, except that the reaction mixture contained radioactive $\beta$-alanine instead of the radioactive pantothenate, and the radioactivity transferred to pantothenate was measured.

III. The rate of the reverse reaction was assayed in a reaction mixture containing D-pantoyl lactone (10-50 mM); $\beta$-alanine (1-10 mM); $\beta-\left[1{ }^{14} \mathrm{C}\right]$ alanine, $5000 \mathrm{~Bq}$ per $100 \mu \mathrm{l}$; the buffer as above; and the enzyme. The radioactivity transferred to pantothenate was counted.

IV. In the determination of the equilibrium constant the reaction mixture contained pantothenate $(5 \mathrm{mM}) ; \beta$-alanine $(5$ or $10 \mathrm{mM})$; pantoyl lactone $(10$ or $25 \mathrm{mM}) ; \beta$ $\left[{ }^{14} \mathrm{C}\right]$ alanine, $5000 \mathrm{~Bq}$ per $100 \mu \mathrm{l}$; the buffer; and the enzyme. The reaction mixtures were incubated overnight, and the radioactivities of both pantothenate and $\beta$-alanine spots were counted.

Thermodynamic parameters. The following ordinary dependences were used to calculate the thermodynamic parameters: $\Delta G=-R T \ln K ; \Delta H=-R \times$ slope (van't Hoff plot $) ; \Delta S=(\Delta H-\Delta G) / T ; \Delta G^{*}=-R T \ln k+73030$ $\left(\mathrm{J} \mathrm{mol}{ }^{-1}\right) ; \Delta H^{\ddagger}=E-R T ; E=-R \times$ slope (Arrhenius plot $) ; \Delta S^{\ddagger}=\left(\Delta H^{\ddagger}-\Delta G^{\ddagger}\right) / T$. In the complex constants $k_{+2} / K_{\mathrm{S}}$ and $K_{\mathrm{B}} k_{+4} / k_{-2}$, the individual rate constants were multiplied or divided by each other such that no sum terms exist. Therefore the logarithms of the rate constants are additive, and further more, the complex constants give meaningful thermodynamic parameters and linear Arrhenius or van't Hoff plots.

Error estimates. The standard deviations for the $\Delta H$ values were calculated from the measured values of the constants by calculating the standard deviations of the slopes of the regression lines of Arrhenius or van't Hoff plots.

The standard deviations of the constants $K_{\mathrm{B}} k_{+4} / k_{-2}$ and $k_{+2} / K_{\mathrm{S}}$ were calculated by simulating 60 series of experiments with five pantothenate and five $\beta$-alanine concentrations, where a standard deviation of the square root of the total counts plus a preparation error of $2 \%$ was given to the calculated isotope counts. The standard deviation of the sixty values of the constants thus derived was expressed.

Theory. The derivation of the kinetic equations is based on the mechanism given in equation (1) in which the binding and dissociation of the components D-pantothenate (S), $\beta$-alanine (B), and D-pantoyl lactone (P) is assumed to be fast enough to be essentially in equilibrium.

$$
\mathrm{E}+\mathrm{S} \underset{K_{\mathrm{S}}}{\underset{k_{-2}}{\rightleftharpoons}} \mathrm{ES} \underset{k_{+2}}{\stackrel{k_{2}}{\rightleftharpoons}} \mathrm{EP}: \mathrm{B} \stackrel{K_{\mathrm{B}} \stackrel{\mathrm{B}}{\longleftrightarrow}}{\leftrightarrows} \mathrm{EP} \underset{k_{-4}}{\stackrel{K_{+4}}{\rightleftharpoons}} \mathrm{E}: \mathrm{P} \stackrel{K_{\mathrm{P}}}{\rightleftharpoons} \mathrm{E}+\mathrm{P}
$$

At first a covalent intermediate EP was assumed to be formed and B dissociates from the enzyme. EP then deacylates and forms free $E$ and $P$. The steady-state assumption and the equilibria lead to a set of five equations (2).

$$
\begin{aligned}
& K_{\mathrm{S}}=\frac{[\mathrm{E}][\mathrm{S}]}{[\mathrm{ES}]} K_{\mathrm{B}}=\frac{[\mathrm{EP}][\mathrm{B}]}{[\mathrm{EP}: \mathrm{B}]} K_{\mathrm{P}}=\frac{[\mathrm{E}][\mathrm{P}]}{[\mathrm{E}: \mathrm{P}]} \\
& k_{+2}[\mathrm{ES}]+k_{-4}[\mathrm{E}: \mathrm{P}]=k_{-2}[\mathrm{EP}: \mathrm{B}]+k_{+4}[\mathrm{EP}] \\
& e=[\mathrm{E}]+[\mathrm{ES}]+[\mathrm{EP}: \mathrm{B}]+[\mathrm{EP}]+[\mathrm{E}: \mathrm{P}]
\end{aligned}
$$

I. The forward reaction rate was measured, using $\left[1-{ }^{14} \mathrm{C}\right]$ pantothenate, by counting the radioactivity of the $\beta$-alanine. At initial rate conditions no radioactivity is returned to $\mathrm{S}$, then the measured rate $v=k_{+2}[\mathrm{ES}]$. (In the presence of non-radioactive $\beta$-alanine this rate is not the rate of pantothenate decomposition which is $k_{+4}[\mathrm{EP}]$.) If no $\mathrm{P}$ is present, $k_{-4}[\mathrm{E}: \mathrm{P}]=0$. The rate equation (3) can then be derived where

$$
v=\frac{k_{+2} e}{1+\frac{\left(1+\frac{K_{\mathrm{B}}}{b}\right) k_{+2}}{k_{-2}+\frac{K_{\mathrm{B}}}{b} k_{+4}}+\frac{K_{\mathrm{S}}}{s}}
$$

$s$ and $b$ are the concentrations of pantothenate and $\beta$ alanine, respectively, and $e$ is the total enzyme concentration. In the form $s / v=f(s)$ (the Hanes plot) the equation is

$\frac{s}{v}=\frac{1+\left(1+\frac{K_{\mathrm{B}}}{b}\right) k_{+2} /\left(k_{-2}+\frac{K_{\mathrm{B}}}{b} k_{+4}\right)}{k_{+2} e} s+\frac{K_{\mathrm{S}}}{k_{+2} e}$.

The direct lines intersect at the ordinate axis, and the intersection point gives $K_{\mathrm{S}} /\left(k_{+2} e\right)$. When $b=0$ the slope is

$\frac{1}{e}\left(\frac{1}{k_{+2}}+\frac{1}{k_{+4}}\right)$.

If the binding and dissociation of the substrate is not much faster than the second step $\left(k_{+2}\right.$ and $\left.k_{-2}\right)$, the lines $s / v=f(s)$ intersect at negative $s$ values. (The equations are not shown.)

II. The isotope-exchange reaction was measured using radioactive $\beta$-alanine, and the radioactivity in pantothenate was counted. The rate of formation of radioactive panto- 
thenate is $v=k_{-2}[\mathrm{EP}: \mathrm{B}]$, and the radioactivity is not returned. (These initial rate conditions hold for a relatively long time since non-radioactive pantothenate must always be present.) The rate equation is:

$$
v=\frac{k_{-2} e}{1+\frac{k_{-2}}{k_{+2}}\left(1+\frac{K_{\mathrm{S}}}{s}\right)+\left[\left(1+\frac{K_{\mathrm{S}}}{s}\right) \frac{k_{+4}}{k_{+2}}+1\right] \frac{K_{\mathrm{B}}}{b}}
$$

and in the form $b / v=f(b)$ the equation is:

$$
\begin{aligned}
\frac{b}{v}= & \left(\frac{1}{k_{-2}}+\frac{1}{k_{+2}}+\frac{K_{\mathrm{S}}}{k_{+2}} \frac{1}{s}\right) \frac{b}{e}+ \\
& {\left[\left(\frac{1}{k_{+2}}+\frac{1}{k_{+4}}\right)+\frac{K_{\mathrm{S}}}{k_{+2}} \frac{1}{s}\right] \frac{K_{\mathrm{B}} k_{+4}}{k_{-2} e} }
\end{aligned}
$$

The intersection point $(x, y)$ of the lines of eqn. (6) at different concentrations of $S$ is in eqn. (7),

$$
\left(b=-\frac{K_{\mathrm{B}} k_{+4}}{k_{-2}} ; \frac{b}{v}=\left(\frac{1}{k_{+4}}-\frac{1}{k_{-2}}\right) \frac{K_{\mathrm{B}} k_{+4}}{k_{+2} e}\right)
$$

where the $x$-value gives the constant $K_{\mathrm{B}} k_{+4} / k_{-2}$ and

$y / x=\left(\frac{1}{k_{+4}}-\frac{1}{k_{-2}}\right) \frac{1}{e}$.

A secondary plot of the slopes of the lines of eqn. (6) against $1 / s$ gives a slope $K_{\mathrm{S}} / k_{+2}$ and an intercept at the ordinate axis of $\left[\left(1 / k_{-2}\right)+\left(1 / k_{+2}\right)\right] 1 / e$. If the association and dissociation steps of $\beta$-alanine to and from the enzyme are not much faster than the deacylation step $\left(k_{+4}\right)$, the expression $b / v=f(b)$ is not a straight line but a hyperbola.

III. The rate of the reverse reaction can be measured if $\beta-\left[{ }^{14} \mathrm{C}\right]$ alanine and pantoyl lactone are present in the reaction mixture, and the formation of $\left[{ }^{14} \mathrm{C}\right]$ pantothenate is measured. The rate is $v=k_{-2}[\mathrm{EP}: \mathrm{B}]$. The rate equation (8) describes this case

$$
v=\frac{k_{-2} e}{\left[1+\left(1+\frac{K_{\mathrm{P}}}{p}\right) \frac{k_{-2}}{k_{-4}}\right]+\left[1+\left(1+\frac{K_{\mathrm{P}}}{p}\right) \frac{k_{+4}}{k_{-4}}\right] \frac{K_{\mathrm{B}}}{b}}
$$

or

$$
\begin{aligned}
\frac{b}{v}= & {\left[\frac{1}{k_{-2} e}+\left(1+\frac{K_{\mathrm{P}}}{p}\right) \frac{1}{k_{-4} e}\right] b+} \\
& {\left[\frac{1}{k_{+4}}+\left(1+\frac{K_{\mathrm{P}}}{p}\right) \frac{1}{k_{-4}}\right] \frac{K_{\mathrm{B}} k_{+4}}{k_{-2} e} }
\end{aligned}
$$

$p$ is the concentration of pantoyl lactone. The intersection point of the lines (9) at different concentrations of $S$ is given by eqn. (10).
$\left(b=-\frac{K_{\mathrm{B}} k_{+4}}{k_{-2}} ; \frac{b}{v}=\left(\frac{1}{k_{+4}}-\frac{1}{k_{-2}}\right) \frac{K_{\mathrm{B}} k_{+4}}{k_{-2} e}\right)$.

The slope $(z)$ of the lines (9) can be plotted as a secondary plot

$z p=\left(\frac{1}{k_{-2} e}+\frac{1}{k_{-4} e}\right) p+\frac{K_{\mathrm{P}}}{k_{-4} e}$

The term $K_{\mathrm{P}} /\left(k_{-4} e\right)$ comes from the intercept of the line and the ordinate axis. If $k_{-2} \gg k_{-4}$, an approximate value of $k_{-4}$ is derived from the slope of the secondary plot (11). In this case $\left(k_{-2} \gg k_{-4}\right)$ the equilibrium constant $K_{\mathrm{P}}$ comes from the intersection point of the line and the abscissa axis.

If the dissociation of $P$ is not a fast step, the intersection point of the lines $b / v=f(b)$ is:

$$
\begin{aligned}
b= & -\left[\frac{k_{+5}}{k_{-4}} /\left(1+\frac{k_{+5}}{k_{-4}}\right)\right] \frac{K_{\mathrm{B}} k_{+4}}{k_{-2}} \\
\frac{b}{v}= & {\left[\left(\frac{1}{k_{+4}}-\frac{1}{k_{-2}}\right)+\right.} \\
& \left.\left(\frac{1}{k_{-4}}+\frac{1}{k_{-2}}\right) /\left(1+\frac{k_{+5}}{k_{-4}}\right)\right] \frac{K_{\mathrm{B}} k_{+4}}{k_{-2}}
\end{aligned}
$$

where $k_{+5}$ is the rate constant for the dissociation of $\mathrm{P}$. (The details of the equations are not shown.) The abscissa value of this intersection point is lower in absolute terms than the corresponding value from the lines (8).

IV. In order to calculate the equilibrium constant, the reaction was allowed run to completion. The radioactivity from $\beta-\left[{ }^{14} \mathrm{C}\right]$ alanine was equilibrated between $\beta$-alanine and pantothenate with same specific activity. By counting the radioactivities in $\mathrm{S}$ and B we obtained $s / b=\alpha$. If $y \mathrm{mM}$ of the substrate has decomposed, then $s=s_{0}-y, b=b_{0}+y$, $p=p_{0}+y$.

Then

$\alpha=\frac{s_{0}-y}{b_{0}+y}$ and $y=\frac{s_{0}-\alpha b_{0}}{1+\alpha}$

$K_{\mathrm{eq}}=\frac{b p}{s}=\frac{1}{\alpha}\left(p_{0}+y\right)=\frac{1}{\alpha} \frac{\left(p_{0}+\alpha p_{0}+s_{0}-\alpha b_{0}\right)}{1+\alpha}$

Another way of calculating the equilibrium constant is to use the rate and dissociation constants in the intermediate steps according to the Haldane relationship.?

$K_{\text {eq }}=\frac{1}{K_{\mathrm{S}}} \frac{k_{+2}}{k_{-2}} K_{\mathrm{B}} \frac{k_{+4}}{k_{-4}} K_{\mathrm{P}}=\frac{k_{+2}}{K_{\mathrm{S}}} \frac{K_{\mathrm{B}} k_{+4}}{k_{-2}} \frac{K_{\mathrm{P}}}{k_{-4}}$ 


\section{Results and discussion}

The total procedure of the kinetic and thermodynamic analysis of the pantothenase-catalyzed reaction contained four different measurements, as described in the methods. It is not possible to solve all the individual rate and dissociation constants by the steady-state methods. If the enzyme follows the kinetics described above, the expressions $k_{+2} / K_{\mathrm{S}}$ (the second-order rate constant), $K_{\mathrm{B}} k_{+4} / k_{-2}$ (the apparent dissociation constant of $\beta$-alanine), and $K_{\mathrm{P}} / k_{-4}$ can be solved, and the constants $k_{-4}$ and $K_{\mathrm{P}}$ can be obtained if the deacylation is the slowest step of the total reaction. These constants and the equilibrium constants of the total reaction were assayed at different temperatures between 11 and $28^{\circ} \mathrm{C}$ using at first the pantothenase from the bacterial strain UK-1. The thermodynamic parameters $\Delta G, \Delta H$, and $\Delta S$ were calculated. Then the same procedure was repeated with the pantothenase PS-21.

\section{A. Pantothenase from $\mathrm{P}$. fluorescens $U K-1$}

I. The rate of the transfer of radioactivity from pantothenate to $\beta$-alanine was measured, the concentrations of pantothenate and $\beta$-alanine were varied, and the results were plotted as $s / v=f(s)$. Fig. 1 shows this kind of assay at $25^{\circ} \mathrm{C}$. The lines intersect at the ordinate axis, as expected if eqn. (4) is followed. The intersection point gives the value of $K_{\mathrm{S}} /\left(k_{+2} e\right)=92 \pm 2 \mathrm{~min}$, or further $k_{+2} / K_{\mathrm{S}}=$ $1780 \mathrm{~s}^{-1} \mathrm{M}^{-1}$. The slope of the line when [ $\beta$-alanine] $=0$ gives: $k_{\text {cat }}=1 /\left[\left(1 / k_{+2}\right)+\left(1 / k_{-4}\right)\right]=13 \mathrm{~s}^{-1}$. As the concen-

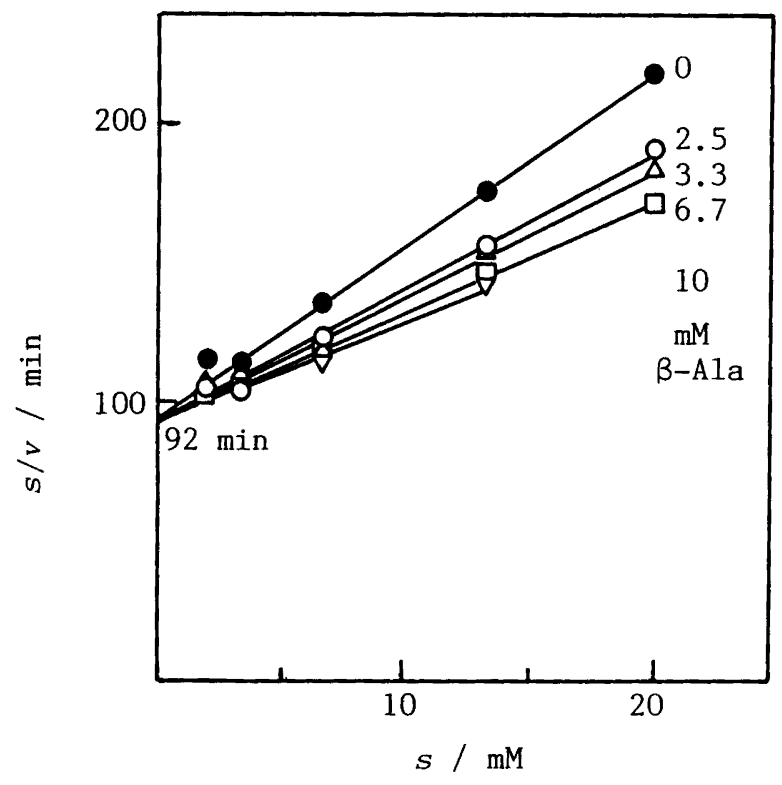

Fig. 1. Pantothenase UK-1-catalyzed transfer of radioactivity from pantothenate to $\beta$-alanine at different concentrations of pantothenate and $\beta$-alanine. The conditions were $25^{\circ} \mathrm{C}, \mathrm{pH} 8.1$, and $e=10.2 \times 10^{-8} \mathrm{M}$.

tration of $\beta$-alanine increases, the slope $1 / V$ decreases $(V$ increases), i.e. the presence of non-radioactive $\beta$-alanine promotes the transfer of radioactivity from pantothenate to $\beta$-alanine. According to eqn. (4) this is the case when $k_{+4}<k_{-2}$.

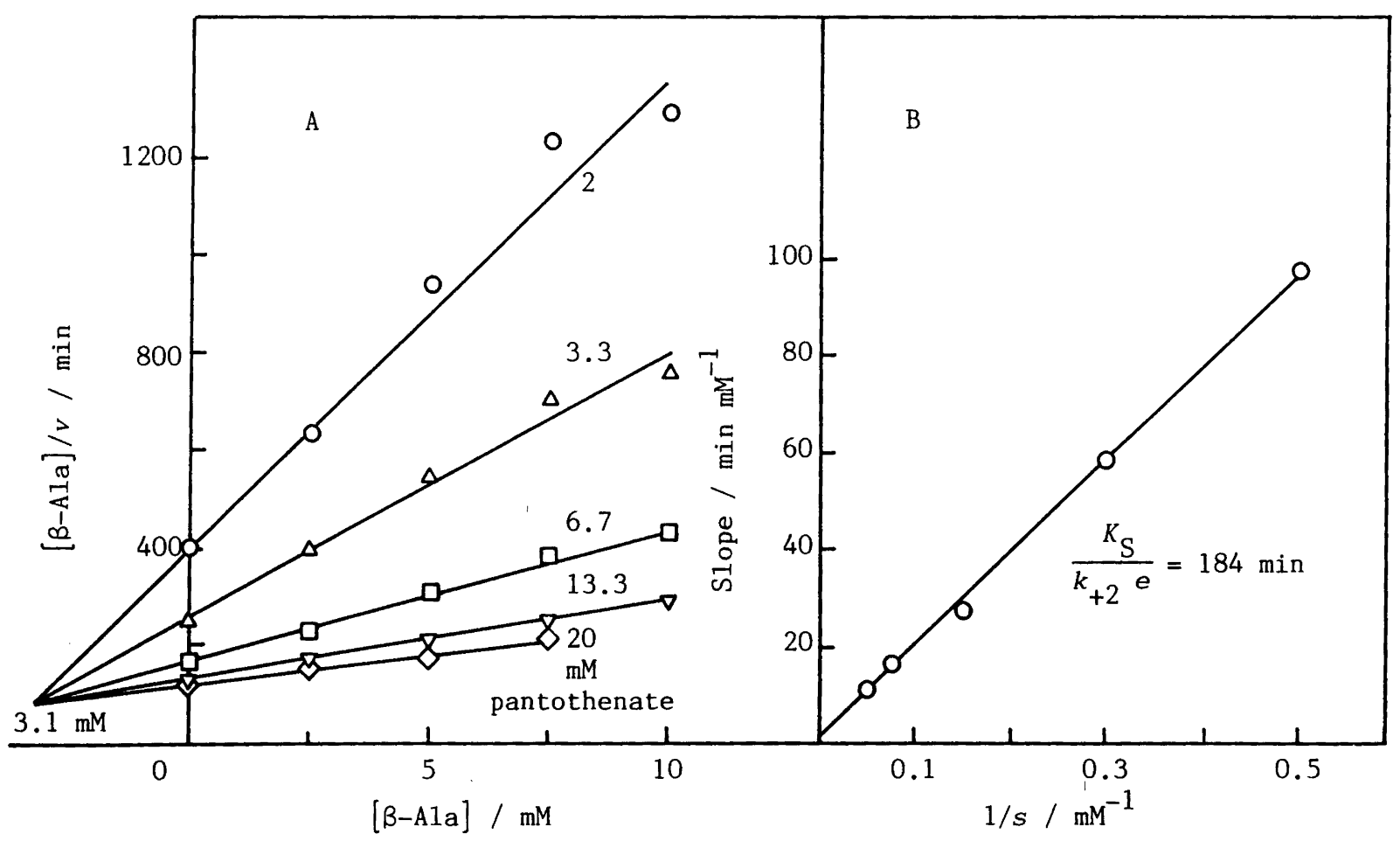

Fig. 2. Pantothenate $-\beta-\left[{ }^{14} \mathrm{C}\right]$ alanine isotope-exchange reaction catalyzed by pantothenase UK-1 at different pantothenate and $\beta$ alanine concentrations at $25^{\circ} \mathrm{C} . e=7.0 \times 10^{-8} \mathrm{M}$. The plots are based on eqn. (6). 


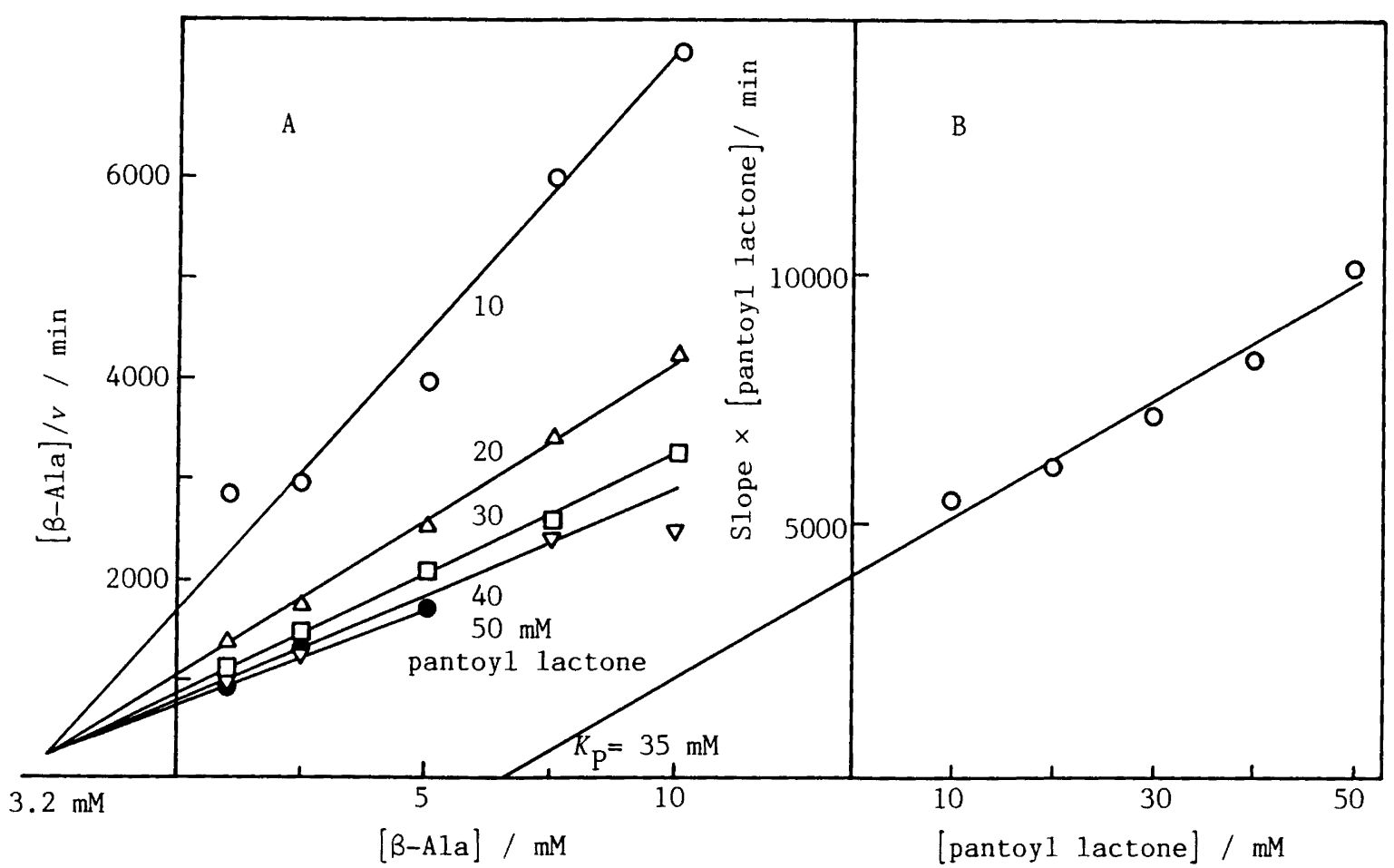

Fig. 3. Formation of pantothenate in the reverse reaction of pantothenase UK-1 at $25^{\circ} \mathrm{C} . e=4.6 \times 10^{-8} \mathrm{M}$. The plots are based on eqns. (8) and (9).

Figs. 4A and 4B show the Arrhenius plots of the $k_{+2} / K_{\mathrm{S}}$ values and the $k_{\text {cat }}$ values derived from assays such a Fig. 1 at different temperatures.

II. Fig. 2 shows an example of an assay of the pantothenate $-\beta-\left[{ }^{14} \mathrm{C}\right]$ alanine isotope-exchange reaction at $25^{\circ} \mathrm{C}$. The concentrations of pantothenate and $\beta$-alanine were varied as in Fig. 1. The intersection point of the lines in Fig. $2 \mathrm{~A}$ gives a value of $K_{\mathrm{B}} k_{+4} / k_{-2}=3.1 \pm 0.23 \mathrm{mM}$. A secondary plot (Fig. $2 \mathrm{~B}$ ) of the slopes against $1 / s$ gives a value of $\left[\left(1 / k_{-2}\right)-\left(1 / k_{+2}\right)\right]=0.007 \mathrm{~s}$, and the slope of the secondary plot gives $K_{\mathrm{S}} / k_{+2}=0.65 \times 10^{-3} \mathrm{~s} \mathrm{M}$. The van't Hoff plot for the values $K_{\mathrm{B}} k_{+4} / k_{-2}$ and the Arrhenius plot for the values $k_{+2} / K_{\mathrm{S}}$ are shown in Figs. $4 \mathrm{~B}$ and $\mathrm{C}$.

The value of $\left[\left(1 / k_{+4}\right)+\left(1 / k_{+2}\right)\right]=0.077 \mathrm{~s}$ from Fig. 1 is much higher than the value of $\left[\left(1 / k_{+2}\right)+\left(1 / k_{-2}\right)\right]=0.007 \mathrm{~s}$ from Fig. 2B. From these values it can be deduced that $k_{+4}$ is less than a tenth of the lower of $k_{+2}$ or $k_{-2}$. Therefore only a small error is made if the $k_{\text {cat }}$ values from Fig. $4 \mathrm{~A}$ are used for $k_{+4}$ later in Fig. 5 (or as well if the values derived from Fig. 3B are used for $k_{-4}$ and $K_{\mathrm{P}}$ ).

III. The rate of the reverse reaction or the formation of pantothenate from $\beta$-alanine and pantoyl lactone (at $\left.25^{\circ} \mathrm{C}\right)$ was measured in Fig. 3. The intercept of the lines [eqn. (9)] gives $K_{\mathrm{B}} k_{+4} / k_{-2}=3.2 \pm 1.6 \mathrm{mM}$. From the secondary plot [eqn. (11)] the approximate values of $K_{\mathrm{P}}=35 \mathrm{mM}$ and $k_{-4}=3.1 \mathrm{~s}^{-1}$ are obtained. (This presupposes that the deacylation is the slowest step as detected above.) Figs. 4C-E show the corresponding Arrhenius and van't Hoff plots when the assays were carried out at different temperatures.

IV. The equilibrium constants were assayed in reaction mixtures containing pantothenate, $\beta$-alanine and pantoyl lactone, and $\beta-\left[{ }^{14} \mathrm{C}\right]$ alanine as the isotope. The reaction was allowed to run overnight, the equilibrium was attained within about $6 \mathrm{~h}$. Fig. 4G shows the van't Hoff plot for the

Table 1. Thermodynamic parameters for the UK-1 enzyme from the Arrhenius and van't Hoff plots in Fig. 4 . The Gibbs energy and entropy values at $25^{\circ} \mathrm{C}$ were calculated. The rate constants were expressed per subunit by dividing the values from Fig. 4 by two.

\begin{tabular}{llllr}
\hline Constant & Value at $25^{\circ} \mathrm{C}$ & $\Delta G$ or $\Delta G^{*} / \mathrm{kJ} \mathrm{mol}^{-1}$ & $\Delta H$ or $\Delta H^{*} / \mathrm{kJ} \mathrm{mol}^{-1}$ & $\Delta S$ or $\Delta G^{*} / \mathrm{J} \mathrm{K}^{-1} \mathrm{~mol}^{-1}$ \\
\hline$K_{+2} / K_{\mathrm{S}}$ & $888 \mathrm{~s}^{-1} \mathrm{M}^{-1}$ & 56 & $26 \pm 3$ & -102 \\
$K_{\mathrm{B}} K_{+4} / K_{-2}$ & $27 \times 10^{-8} \mathrm{M}$ & 26 & $21 \pm 3$ & -16 \\
$K_{\text {cat }}\left(\approx K_{+4}\right)$ & $6.8 \mathrm{~s}^{-1}$ & 68 & $29 \pm 3$ & -133 \\
$K_{-4}$ & $1.6 \mathrm{~s}^{-1}$ & 72 & $37 \pm 4$ & -118 \\
$K_{\mathrm{p}}$ & $35 \times 10^{-3} \mathrm{M}$ & 8.6 & $24 \pm 3$ & +51 \\
$K_{\text {eq }}$ (measured) & $0.71 \times 10^{-3} \mathrm{M}$ & 18 & $37 \pm 5$ & +65 \\
$K_{\text {eq }}$ (calc.) & $0.47 \times 10^{-3} \mathrm{M}$ & 19 & $36 \pm 4$ & +58 \\
\hline
\end{tabular}




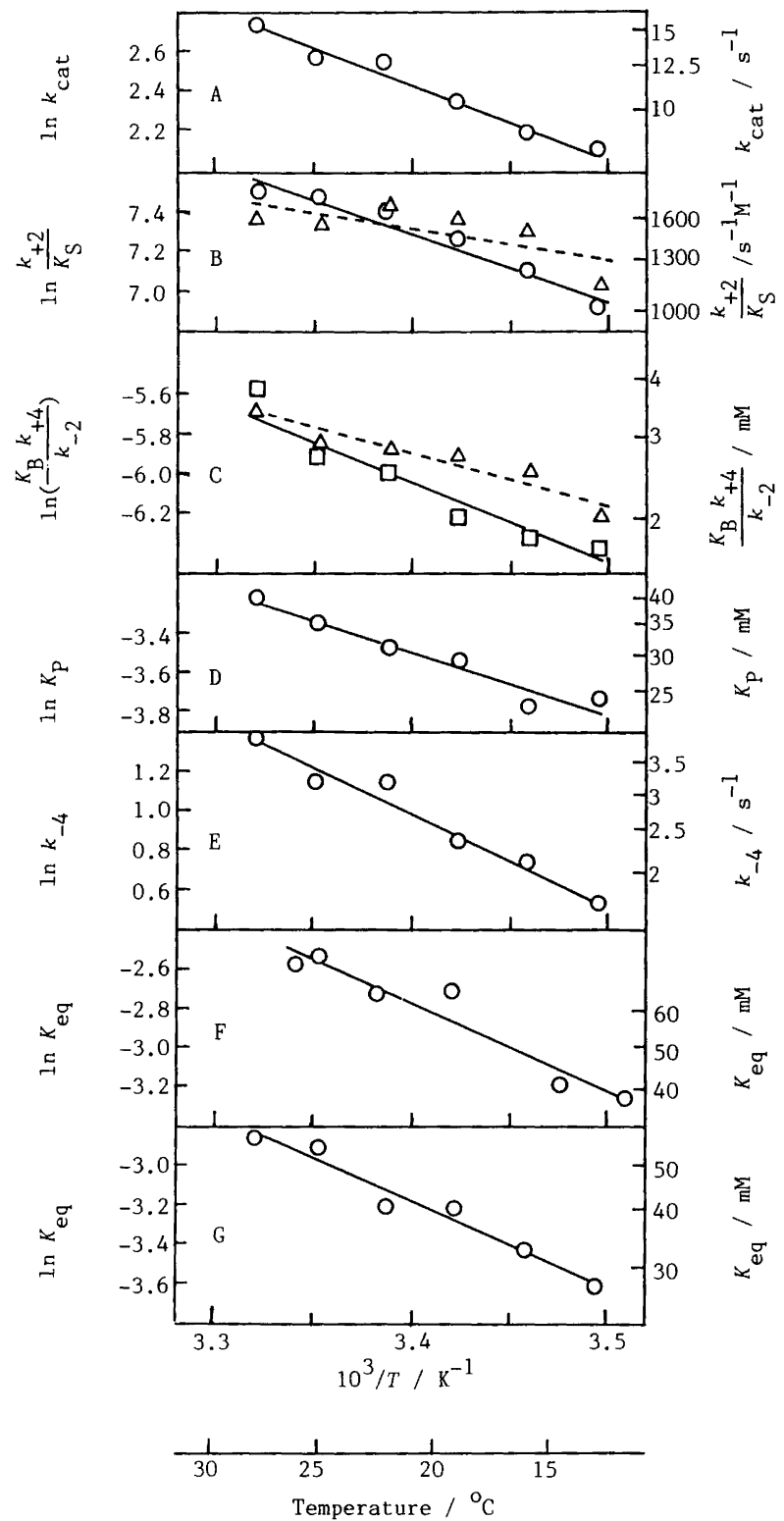

Fig. 4. Arrhenius plots (A, B, E) and van't Hoff plots (C, D, F, G) of the kinetic constants obtained by measurements of the pantothenase UK-1 reactions at different temperatures. A, the $k_{\text {cat }}$ values are from type I measurements (e.g. Fig. 1); B, the $k_{+2} / K_{\mathrm{S}}$ values are from type I $\left(\mathrm{O}_{-}-\mathrm{O}\right.$, Fig. 1$)$ or type II ( $\triangle \cdots \triangle$, Fig. 2) measurements; $C$, the $K_{\mathrm{B}} k_{+4} / k_{-2}$ values are from type II $(\triangle \cdots \triangle)$ or type III ( $\square-\square$, Fig. 3) assays. D and $\mathrm{E}$, the $K_{\mathrm{P}}$ and $k_{-4}$ values are from type III assays. $F$, the measured $K_{\mathrm{eq}}$ values, and $\mathrm{G}$, the calculated $K_{\mathrm{eq}}$ values where the constants from $B(O), C(\triangle), D$, and $E$ were used.

equilibrium constants obtained in such measurements at different temperatures.

The values of the kinetic constants from Figs 4B, C, D, and $\mathrm{E}$ were used to calculate the equilibrium constants according to eqn. (15). The van't Hoff plot of these constants is shown in Fig. 4G.

In Table 1 the slopes in Fig. 4 were used to calculate the $\Delta H$ and $\Delta H^{\ddagger}$ values. The $\Delta G$ and $\Delta G^{\ddagger}$ values were de-

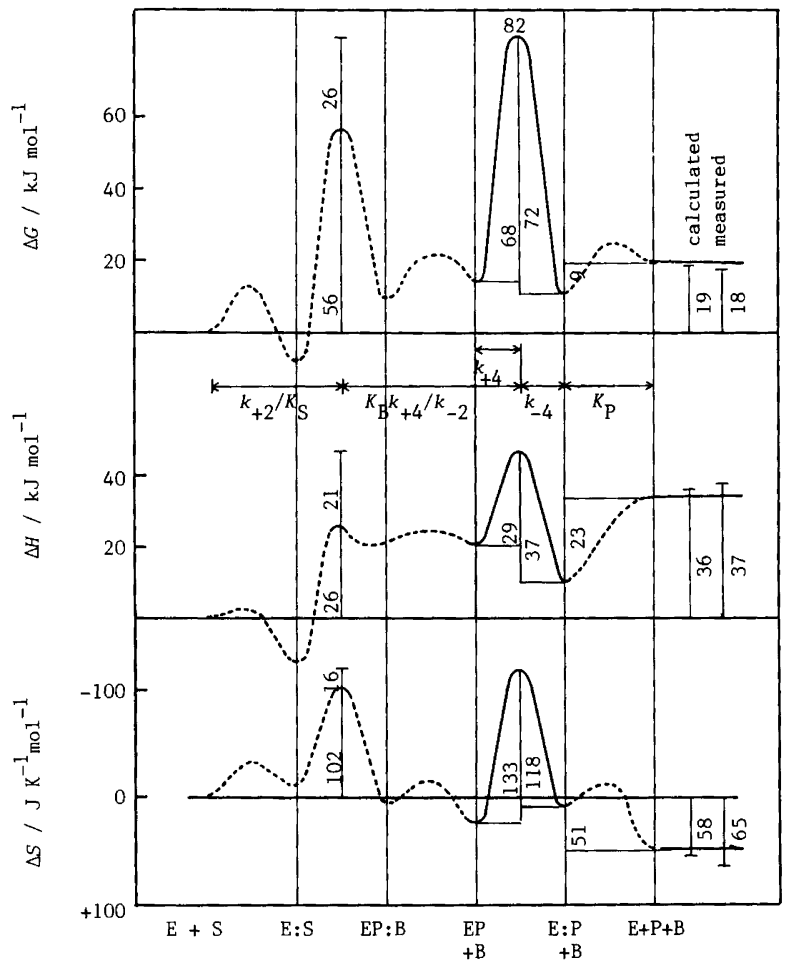

Fig. 5. The energy profiles for the pantothenate decomposition by pantothenase UK-1. The thermodynamic parameter values are from Table 1.

rived from the rate and dissociation constant values at $25^{\circ} \mathrm{C}$ (the values from the regression lines were used). The $\Delta G^{\neq}$ values were calculated per subunit; the pantothenase UK-1 contains two subunits of same size. ${ }^{2}$ The $-\mathrm{NH}_{2}$ form of $\beta$-alanine reacts in the reverse reaction, ${ }^{4}$ therefore the quantity $K_{\mathrm{B}} k_{+4} / k_{-2}$ was also calculated to correspond to the concentration of the non-protonated amino group when calculating the $\Delta G$ value. The $\mathrm{p} K$ value of the amino group is 10.25 , thus the value of $K_{\mathrm{B}} k_{+4} / k_{-2}$ from Fig. 4 was divided by 108 .

Finally the calculated values of the thermodynamic constants were used in drawing the energy profiles in Fig. 5.

\section{B. Pantothenase from Pseudomonas PS-21}

Fig. 6 shows the measurements of the forward reaction and Fig. 7 the isotope-exchange reaction of pantothenase PS-21 at $25^{\circ} \mathrm{C}$. The results are very similar to the results with the other enzyme in Figs. 1 and 2. The transfer of radioactivity from pantothenate to $\beta$-alanine is increased in the presence of non-radioactive $\beta$-alanine, and the intersection points of the lines are at the corresponding sites. The values of the constants from Figs. 6 and 7 are: $k_{+2} / K_{\mathrm{S}}=4720 \mathrm{~s}^{-1} \mathrm{M}^{-1}$, $k_{\text {cat }}=28 \mathrm{~s}^{-1}$, and $K_{\mathrm{B}} k_{+4} / k_{-2}=9.3 \pm 0.7 \mathrm{mM}$. As described above, it can be deduced that $k_{+4}$ is less than a seventh of the lower of $k_{+2}$ or $k_{-2}$. The constants assayed at different temperatures give the Arrhenius and van't Hoff plots of Fig. 8. The calculated thermodynamic constants are in 


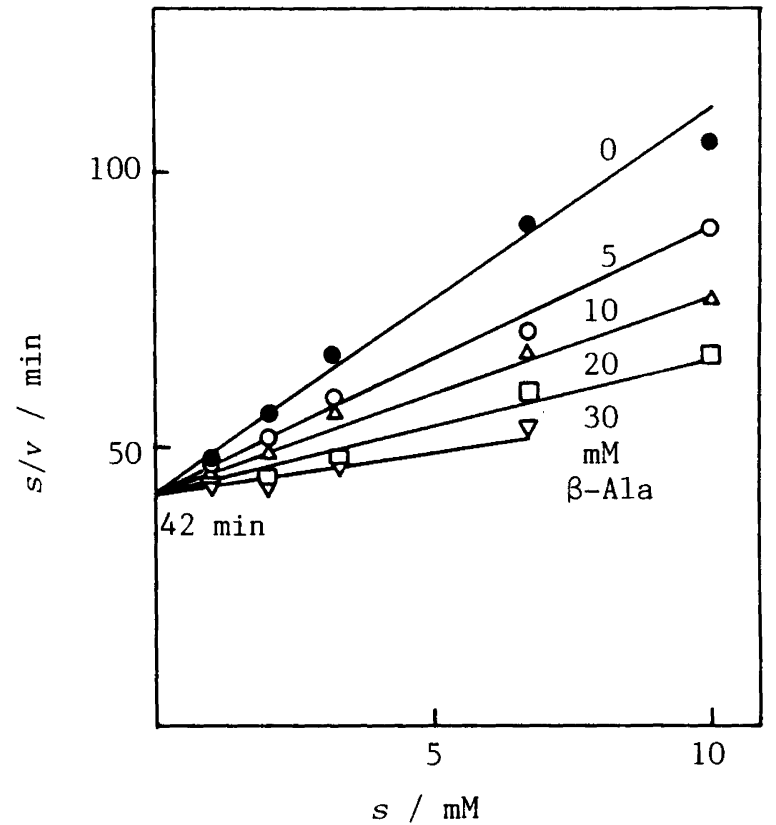

Fig. 6. Rates of the forward reaction of pantothenase PS-21. The assay corresponds to Fig. 1. $e=8.4 \times 10^{-8} \mathrm{M}$.

Table 2. As in Table 1, the constants were calculated per subunit and the $-\mathrm{NH}_{2}$ form of $\beta$-alanine. The enzyme contains four subunits (the number of active sites is not yet known). ${ }^{3}$

The $\mathrm{p} K$ value of the carboxy group of the pantoic acid is 4.2 ; at $\mathrm{pH} 8.1$ pantoic acid is almost totally in the deprotonated form. The dissociation of the proton shifts the equilibrium of the pantothenate hydrolysis far to the side of the products. Therefore it is not possible to measure the reverse reaction in the way described for pantothenase UK-1. The reverse reaction was measured using a high concentration of pantoate $(100 \mathrm{mM})$, a relatively low concentration of $\beta$-alanine ( $2 \mathrm{mM}$, in order to keep the specific radioactivity high), and no added pantothenate. Even under these conditions the radioactivity transferred to pantothenate $(0.6 \mathrm{~Bq})$ was lower than the background radioactivity in this method $(1.3 \mathrm{~Bq})$, therefore no accurate measurements were possible. An estimate of the equilibrium constant thus attained is $42 \mathrm{M}$. The difference between the equilibrium constants of the pantothenate decomposition in the two enzymic reactions is affected both by the dissociation of the pantoic acid $(\mathrm{p} K=4.2)$ and the equilibrium between pantoic acid and pantoyl lactone. When the measured values are used, the calculation of the equilibrium constant gives $K=$ [pantoyl lactone]/[pantoic acid $]=15$. This value seems low compared with the formation constants of lactones from $\gamma$-hydroxybutyric acid (2.7) or $\gamma$-hydroxyvaleric acid (13.9); ${ }^{8}$ the two methyl groups at $\beta$-position in the pantoic acid apparently force the $\gamma$-hydroxy group close to the carboxy group.

The first point raised by the results is the correctness of eqn. (1) for both enzymes, i.e. the acyl enzyme mechanism and the use of the dissociation constants $K_{\mathrm{S}}, K_{\mathrm{B}}$ and $K_{\mathrm{P}}$ instead of the corresponding expressions by rate constants. The evidence for the acyl enzyme mechanism includes (1) the existence of the pantothenate $-\beta-\left[{ }^{14} \mathrm{C}\right]$ alanine isotopeexchange reaction as in Figs. 2 and $7 ;(2)$ the dependence of the transfer of radioactivity from pantothenate to $\beta$-alanine on the $\beta$-alanine concentration (Figs. 1 and 6); (3) the almost identical $k_{+2} / K_{\mathrm{S}}$ and $K_{\mathrm{B}} k_{+4} / k_{-2}$ values obtained from different measurements (Figs. 4 and 8); and (4)

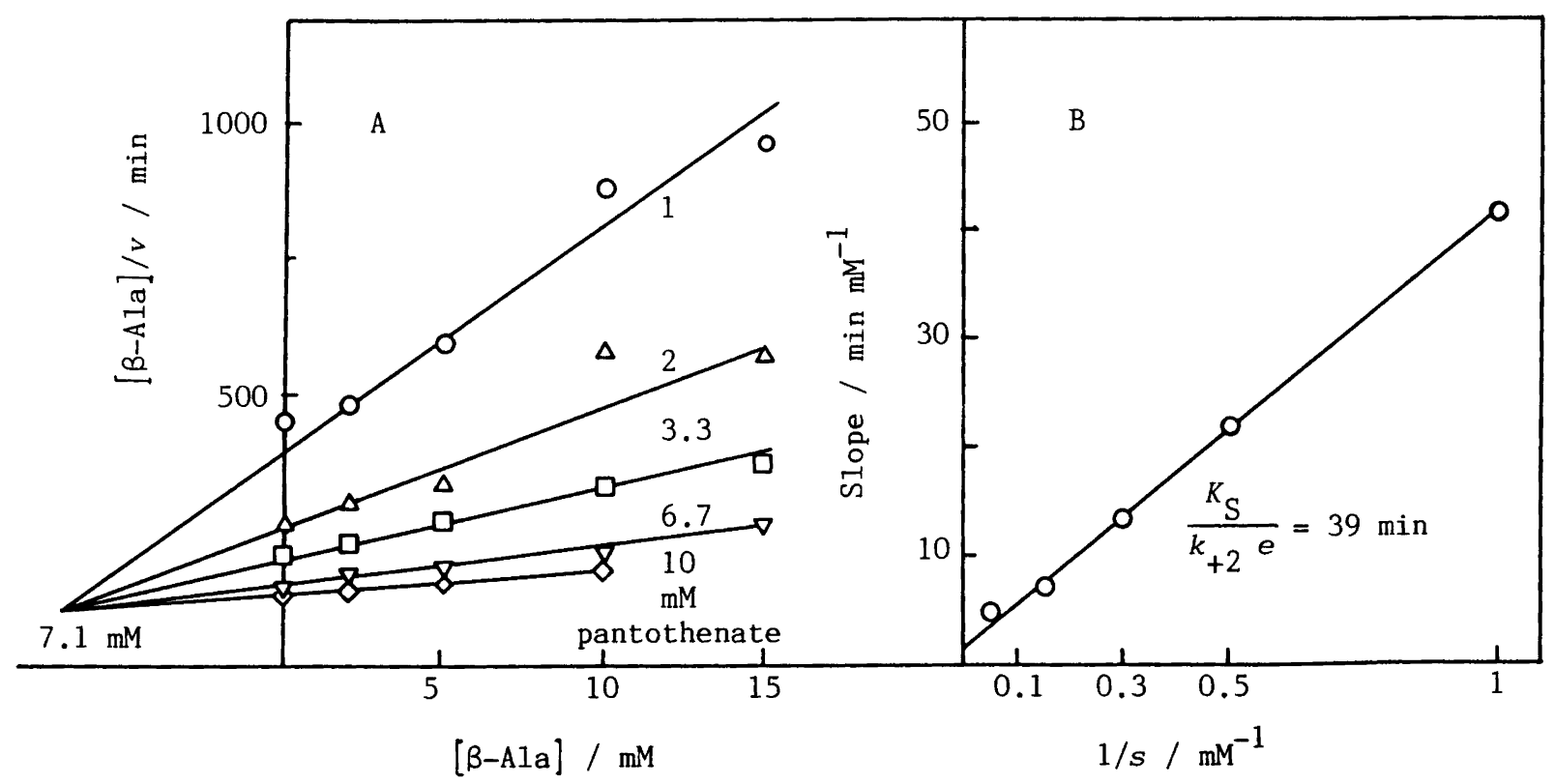

Fig. 7. Rates of the isotope-exchange reaction of pantothenase PS-21 at different pantothenate and $\beta$-alanine concentrations. The assay corresponds to Fig. $2 . e=5.6 \times 10^{-8} \mathrm{M}$. 


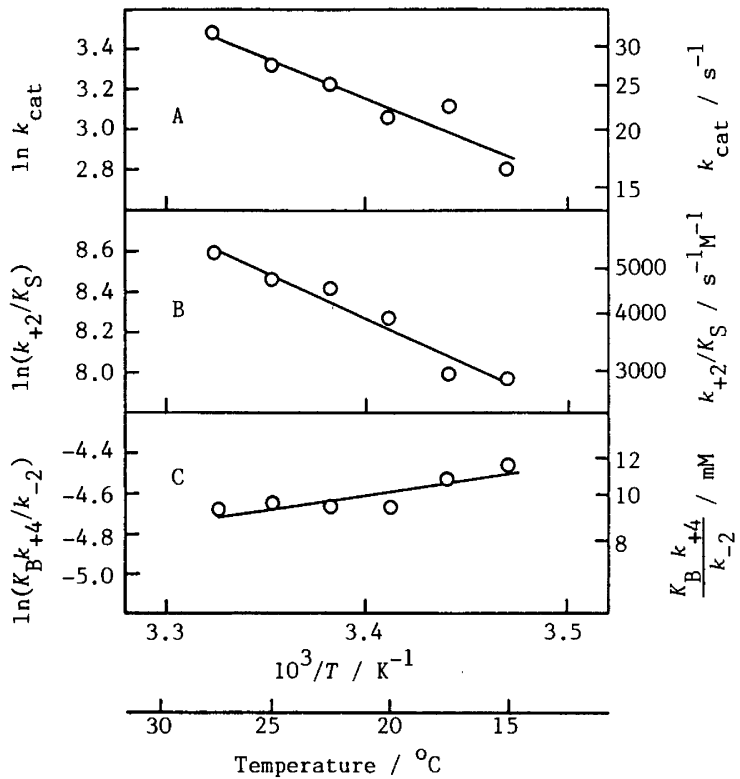

Fig. 8. Arrhenius (A, B) and van't Hoff (C) plots of the kinetic constants of the pantothenase PS-21 reaction at different temperatures. The values of the constants in $A$ and $B$ are from type I and in C from type II assays.

8); and (4) the very similar calculated and measured equilibrium constant values (Figs. $4 \mathrm{~F}$ and $\mathrm{G}$ ). These features were similar in both pantothenases (except the $K_{\text {eq }}$ of pantothenase PS-21 which could not be measured).

The results also show that within the limits of accuracy of the measurements, the dissociation constants of the substrate and products can be used instead of the corresponding rate constants. These steps should be faster than the acylation and deacylation steps in order to be essentially in equilibrium. The evidence for this includes: (1) The intersection point in Figs. 1 and 6 fall on the ordinate axis. If the binding of pantothenate were a slow step, the intersection point would be at negative abscissa values; (2) the lines in Figs. $2 \mathrm{~A}$ and $7 \mathrm{~A}$ are not systematically curved. If the dissociation of $\beta$-alanine were a slow step, the plots should be curved upwards (theory section part II); (3) about the same value of the quantity $K_{\mathrm{B}} k_{+4} / k_{-2}$ is obtained from Figs. 2 and 3 (Fig. 4C). If the dissociation of pantoate were a slow step, the value from Fig. 3 should be lower than that from Fig. 2 (part III in the Theory section).

The thermodynamic parameters of the pantothenase UK-1 reaction follow the normal pattern of an amidase reaction. In chymotrypsin (as an example of an amidase functioning with the acyl enzyme mechanism), the activation enthalpies of both acylation and deacylation are positive, and the activation entropies and negative; both of them thus contribute to the energy barriers at the transition states. ${ }^{9-12}$ Also, patothenase UK-1 shows positive activation enthalpies and negative activation entropies both in acylation and deacylation.

Pantothenase PS-21 differs from pantothenase UK-1 in that it produces pantoic acid instead of pantoyl lactone. ${ }^{3}$ Water participates in the total deacylation reaction of pantothenase PS-21 but the deacylation of the acyl-pantothenase UK-1 can be written without the participation of water. In the latter, the $\gamma$-hydroxy group of the pantoyl moiety would function as the nucleophile instead of water. Such a difference in the reaction mechanism - if true - could perhaps cause a difference also in the thermodynamic parameters. There is, in fact, a difference in the $\Delta H$ values of the apparent dissociation constants $\left(K_{\mathrm{B}} k_{+4} / k_{-2}\right)$ of $\beta$-alanine from the two enzymes. In pantothenase UK-1 the value is $21 \mathrm{~kJ} \mathrm{~mol}^{-1}$ but in pantothenase PS-21 the expression is slightly lowered with increasing temperature to give a negative $\Delta H$ of $-12 \mathrm{~kJ} \mathrm{~mol}^{-1}$. The difference in the $\Delta H$ seems to reflect a difference in the mechanism, or that the deacylation of the pantoyl-pantothenase UK-1 can occur without the participation of water. The difference is produced before the rate-limiting step (the $\Delta H^{*}$ of the $k_{\text {cat }} \approx k_{+4}$ is positive). If the binding of water is added to eqn. (1) [eqn. (16)]

$\rightleftharpoons \mathrm{EP}: \mathrm{B} \stackrel{K_{\mathrm{B}} \stackrel{\mathrm{B}}{\longrightarrow}}{\rightleftharpoons} \mathrm{EP} \underset{K_{\mathrm{H}_{2} \mathrm{O}}}{\stackrel{\mathrm{H}_{2} \mathrm{O}}{\longrightarrow}} \mathrm{EP}:\left(\mathrm{H}_{2} \mathrm{O}\right) \underset{k_{-4}}{\stackrel{K_{+4}}{\rightleftharpoons}} \mathrm{E}: \mathrm{P} \rightleftharpoons$

then the measured apparent dissociation constant of $\beta$ alanine is expressed by $K_{\mathrm{B}} k_{+4} /\left(k_{-2} K_{\mathrm{H}_{2} \mathrm{O}}\right)$. The temperature dependences of $k_{+4}$ and $k_{-2}$ are not expected to differ much from each other, and $K_{\mathrm{B}}$ and $K_{\mathrm{H}_{2} \mathrm{O}}$ are, most probably, increased with temperature. The $\Delta H$ for the given expression would then be lower than the $\Delta H$ for the corresponding expression $K_{\mathrm{B}} k_{+4} / k_{-2}$ for the pantothenase UK-1.

Water also participates in the deacylation of acyl-chymotrypsin, and the $\Delta H$ between the transition states at acylation and deacylation [corresponding to the $\Delta H$ of the constant $\left.K_{\mathrm{B}} k_{+4} /\left(k_{-2} k_{\mathrm{H}_{2} \mathrm{O}}\right)\right]$ is close to zero in the hydrolysis of the acetyltyrosine ethyl ester. ${ }^{9}$

Table 2. Thermodynamic parameters for the PS-21 enzyme derived from Fig. 8. The calculations were as in Table 1 except that the rate constants in Fig. $8\left(\right.$ at $25^{\circ} \mathrm{C}$ ) were divided by four in order to express them per subunit.

\begin{tabular}{lllrr}
\hline Constant & Value at $25^{\circ} \mathrm{C}$ & $\Delta G$ or $\Delta G^{*} / \mathrm{kJ} \mathrm{mol}^{-1}$ & $\Delta H$ or $\Delta H^{+} / \mathrm{kJ} \mathrm{mol}^{-1}$ & $\Delta S$ or $\Delta G^{+} / \mathrm{J} \mathrm{K}^{-1} \mathrm{~mol}^{-1}$ \\
\hline$K_{+2} / K_{\mathrm{S}}$ & $1200 \mathrm{~s}^{-1} \mathrm{M}^{-1}$ & 55 & $36 \pm 4$ & -67 \\
$K_{\mathrm{B}} K_{+4} / k_{-2}$ & $86 \times 10^{-6} \mathrm{M}$ & 23 & $-12 \pm 4$ & -118 \\
$K_{\text {cat }}\left(\approx K_{+4}\right)$ & $7.1 \mathrm{~s}^{-1}$ & 68 & $32 \pm 5$ & -122 \\
\hline
\end{tabular}




\section{References}

1. Nurmikko, V., Salo, E., Hakola, H., Mäkinen, K. and Snell, E. E. Biochemistry 5 (1966) 399.

2. Airas, R. K. Methods Enzymol. 62 (1979) 267.

3. Airas, R. K. Biochem. J. 250 (1988) 447.

4. Airas, R. K. Anal. Biochem. 134 (1983) 122.

5. Fastrez, J. and Fersht, A. R. Biochemistry 12 (1973) 2025.

6. Ellis, K. J. and Morrison, J. F. Methods Enzymol. 87 (1982) 405.

7. Dixon, M. and Webb, E. C. Enzymes, 3rd ed., Longmans Green, London 1979, pp. 69-70.
8. Jencks, W. P. Catalysis in Chemistry and Enzymology, McGraw-Hill, New York 1969, p. 15.

9. Rajender, S., Han, M. and Lumry, R. J. Am. Chem. Soc. 92 (1970) 1378.

10. Martinek, K., Dorovska, V. N., Varfolomeyev, S. D. and Berezin, I. V. Biochim. Biophys. Acta 271 (1972) 80.

11. Wang, C.-L. A., Calvo, K. C. and Klapper, M. H. Biochemistry 20 (1981) 1401.

12. Laidler, K. J. and Peterman, B. F. Methods Enzymol. 63 (1979) 234.

Received November 21, 1988. 